\title{
A informação dos sistemas de informação gerenciais como elemento determinante no apoio à tomada de decisão em hospitais $^{1}$
}

\author{
Information from managing information systems as a fundamental \\ element to support decision-making in hospitals
}

Cibele Roberta SUGAHARA ${ }^{2}$

José Henrique SOUZA ${ }^{3}$

Joseani VISELI ${ }^{4}$

\section{RESUMO}

Neste artigo abordamos a atividade de determinação das informações de um sistema hospitalar como primordial para a escolha do tipo de sistema de informação a ser utilizado e do nível organizacional a ser apoiado. $\bigcirc$ uso de um sistema de informações gerenciais em hospitais melhora a tomada de decisão ao permitir ao usuário do sistema a geração de relatórios de controle e acompanhamento dos procedimentos realizados no hospital.

Palavras-chave: informação; sistemas de informação; sistemas de informação gerenciais; sistemas hospitalares.

\begin{abstract}
This article deals with the activity of defining information of hospital systems as fundamental for choosing the type of information systems to be used and also the organizational level to be supported. The use of hospital managing information systems improves the user's decision -making process by allowing control report generation and following up the procedures made in the hospital as well.
\end{abstract}

Keywords: information; information systems; managing information systems; hospital systems.

\footnotetext{
Parte deste artigo é oriundo da monografia "O sistema de informações gerenciais e a nova tecnologia hospitalar", apresentado à Faculdade de Ciências Econômicas do Centro de Economia e Administração da PUC Campinas, em dezembro de 2004.

2 Doutorado em andamento em Ciência da Informação, Universidade de São Paulo, USP, Brasil. Docente da Faculdade de Ciências Econômicas, Contábeis e Administrativas, Pontifícia Universidade Católica de Campinas, PUC-Campinas, SP, Brasil. Rod. Dom Pedro I, Km 136, 13086-900, Pq. das Universidades, Campinas, SP, Brasil. <cibelesu@puc-campinas.edu.br>.

3 Doutor em Política Científica e Tecnológica, Universidade Estadual de Campinas, UNICAMP, SP, Brasil. Docente do Centro de Economia e Administração, Pontifícia Universidade Católica de Campinas, PUC-Campinas, SP, Brasil. Rod. Dom Pedro I, Km 136, 13086-900, Pq. das Universidades, Campinas, SP, Brasil. <josehenriquesouza@yahoo.com.br>.

4 Formada em Economia na Pontifícia Universidade Católica de Campinas em 2004. Recebido em 6/7/2007 e aceito para publicação em 31/1/2008.
} 


\section{INTRODUÇÃO}

A diversidade de sistemas existentes para dar suporte às funções e aos processos de negócio de uma organização desperta à premente necessidade de se rever o papel da informação nos tipos de sistemas existentes nas instituições. Os sistemas sozinhos não podem fornecer todas as informações necessárias a uma instituição, por isso o ser humano desempenha papel chave no processo de determinação das informações que irão fazer parte do sistema. Nesse contexto, a definição do sistema a ser utilizado na organização para apoiar os gestores depende, principalmente, das áreas funcionais (recursos humanos, marketing entre outras), e do nível organizacional (estratégico, gerencial, de conhecimento e operacional) que se pretende atender.

Conforme Shannon e Weaver (1962), a informação é aquilo que reduz a incerteza. Segundo Le Coadic (1996, p.5) "a informação é um conhecimento inscrito (gravado) sob a forma escrita (impressa ou digital), oral ou audiovisual." Nesse sentido, a informação também pode ser organizada em sistemas manuais ou automatizados visando apoiar a tomada de decisão. E após a definição das informações que compõem um processo de negócio ${ }^{5}$ deve-se pensar no tipo de sistema que poderá dar suporte a esse processo. Nesse contexto, a informação orientada para atender a um determinado nível da organização determina o tipo de sistema necessário para o seu processamento.

Neste artigo, relatamos como a informação pode ser processada por um sistema de informação gerencial em hospitais, visando integrar as informações das áreas internas ao hospital e dar suporte aos gestores do nível gerencial. Cabe ressaltar que a simples adoção de um sistema de informação não garante a organização e integração de informações. Cabe ao gestor da informação definir quais informações serão organizadas, e integradas com o uso de sistemas tradicionais ou integrados.

\section{SISTEMAS DE INFORMAÇÃO: CONCEITOS E TIPOS} complexa, organizada, que capta, armazena, processa, fornece, usa e distribui informação, incluindo os recursos organizacionais relacionados, tais como recursos humanos, tecnológicos e financeiros. Nesse sentido, é um sistema humano que inclui, provavelmente, recursos computacionais para automatizar determinados elementos do sistema (Robredo, 2003).

Os sistemas de maior complexidade podem ser entendidos como um conjunto de subsistemas, cada um deles mantendo, num nível de complexidade menor, as características sistêmicas. Assim, pode-se dizer que os subsistemas estão inseridos em outros subsistemas. Essa visão pode ajudar no planejamento e gestão de sistemas complexos de informação, associando processos parciais específicos a subsistemas próprios, adequadamente integrados ao conjunto, como, por exemplo, subsistema de análise temática da informação, subsistema de busca e recuperação, entre outros (Robredo, 2003).

Como destaca Robredo (2003), a informação sempre está associada a algum tipo de sistema, é uma invenção do homem, criada com intuito de obter algum benefício ou vantagem e, geralmente, alicerçada em algum tipo de tecnologia avançada.

Conforme Hawkins (2001, p.44-53), "[...] vivemos numa sociedade de informação na qual mais gente deve administrar mais informação, que por sua vez requer mais suporte tecnológico, o qual requer e gera mais informação." A partir dessa definição, podese deduzir que o uso de sistemas pode auxiliar a organização e processamento da informação.

Segundo Oliveira (2001), sistema é um conjunto de partes interagentes e interdependentes que, conjuntamente, formam um todo unitário com determinado objetivo e efetuam determinada função. Seus componentes principais são: a) os objetivos dos usuários do sistema e os objetivos do próprio sistema; b) as entradas do sistema que fornecem o material (dados) a ser aproveitado para a geração das saídas, isto é, as informações necessárias estabelecidas pelos objetivos anteriormente estabelecidos; c) o processo de transformação do sistema definido como a função que possibilita a transformação de um insumo (entrada) em um produto, serviço ou resultado (saída); d) as saídas do sistema, que correspondem aos resultados do processo de transformação; e) os controles e as

\footnotetext{
5 Processos de negócios referem-se aos métodos exclusivos segundo os quais o trabalho é organizado, coordenado e focado para produzir um produto ou serviço de valor. São exemplos de processos de negócio: desenvover um novo produto, gerar um pedido de compra, entre outros (Laudon; Laudon, 2004, p.63).
} 
avaliações do sistema que verificam se as saídas estão coerentes com os objetivos estabelecidos; e f) o feedback do sistema através da re-introdução de uma saída sob a forma de informação.

Os sistemas de informação podem ser classificados segundo o nível organizacional a que atendem em: Sistema de Informações Estratégicas (SIE); Sistema de Informações Gerenciais (SIG); Sistema de Informações de Conhecimento (SIC) e Sistema de Informações Operacionais (SIO).

O Sistema de Informações do nível estratégico apoia a gerência atacando e enfrentando questões estratégicas e tendências de longo prazo no ambiente interno e externo, buscando compatibilizar as mudanças no ambiente externo com a capacidade da organização. Esse tipo de sistema deve estar apto a fornecer informações, por exemplo, sobre as tendências de longo prazo do setor no qual a instituição está inserida e os níveis de emprego em cinco anos, entre outras (Laudon; Laudon, 2004, p.40).

Sistema de Informações do nível gerencial auxilia as atividades de monitoração, controle, tomada de decisões e procedimentos administrativos dos gestores médios, visando atender ao nível gerencial da organização, apoiando a tomada de decisões ao fornecer, por exemplo, relatórios ou acesso on-line aos registros do desempenho corrente e histórico da organização. Esse sistema apoia, por exemplo, as atividades de controle de estoque; análise de investimento de recursos e análise de realocação (Laudon; Laudon, 2004, p.40).

É a partir dos dados agrupados ou sintetizados das operações das funções empresariais da empresa que os SIGs operam, apoiando a tomada de decisão do corpo gestor ou gerencial das unidades departamentais, em sinergia com as demais unidades (Rezende; Abreu, 2003, p. 134).

O Sistema de Informações do nível do conhecimento apoia os trabalhadores do conhecimento e de dados da organização, ajudando a organização na integração de novas tecnologias ao negócio e no controle do fluxo de documentos. As estações de trabalho de projeto científico ou de engenharia possibilitam a criação de novos conhecimentos e asseguram que estes sejam adequadamente integrados à empresa.
Já o Sistema de Informações do nível operacional apoia os gestores operacionais, acompanhando as atividades e transações elementares da organização, respondendo às perguntas de rotina e acompanhando o fluxo de transações pela organização. Além disso, fornece informações sobre atividades elementares, como: contas a pagar, contas a receber, treinamento e desenvolvimento entre outras. Essas informações servirão de apoio às outras atividades, tais como: previsão quinquenal de orçamento; planejamento de lucros; planejamento de pessoal entre outras (Laudon; Laudon, 2004, p.39).

As organizações estão inseridas em um ambiente vasto de informações, disso resultando a necessidade de se fazer a gestão da informação utilizando um sistema como apoio. O SIG pode auxiliar a gestão da informação na organização quando "alimentado" com informações provenientes das áreas funcionais da organização (compras, pesquisa e desenvolvimento, entre outras). Cabe ressaltar que, devido à imensa quantidade de dados existentes nas organizações, fazse necessário selecionar dentre os dados e as informações disponíveis aqueles que de fato devem ser processados por um sistema visando melhorar a tomada de decisão.

Um sistema de informações é composto por um conjunto de dados que entram no sistema e outros conjuntos de dados mantidos em arquivos ou tabelas, e sobre os quais se aplica uma rotina de trabalho, um programa, um processamento, de modo a obtermos informações de saída. O Sistema de Informações Gerenciais (SIG) começa a operar a partir do momento em que os Sistemas de Processamento de Transações (SPT's) fornecem dados resumidos de transações ao sistema de relatórios do SIG, no final de um período de tempo determinado. Os gerentes têm acesso aos dados organizados por meio do SIG que the disponibiliza os relatórios adequados.

Neste artigo daremos enfoque ao Sistema de Informação Gerencial (SIG) relatando sobre o seu uso em hospitais.

\section{SISTEMAS DE INFORMAÇÃO GERENCIAIS EM HOSPITAIS}

A atividade de determinação das informações necessárias para apoiar as pessoas na execução de 
suas tarefas precisa ser desenvolvida antes da escolha do sistema de informação a ser utilizado. Assim, os sistemas formais de informação não precisam adaptarse às necessidades dos usuários no fornecimento de dados importantes.

A falta de clareza na definição das informações que farão parte do sistema dos hospitais pode acarretar inúmeros transtornos aos usuários, contribuindo com a má qualidade da assistência prestada. Conforme Davenport (1998), determinar as exigências da informação é uma tarefa difícil, pois envolve identificar como as pessoas percebem seus ambientes informacionais. Os analistas da informação, ao acompanharem de perto os gestores, poderão entender desde o princípio as tarefas administrativas e as necessidades informacionais, conhecendo, por meio das observações, a informação estruturada e não estruturada, a formal e a informal, e definindo as informações que devem compor o sistema.

Conforme relatado em trabalho anterior de Souza; Viseli e Sugahara (2006), os transtornos mais comuns resultantes da falta da determinação e organização das informações em um sistema que possa apoiar a tomada de decisão são: a) informações desatualizadas que não contribuem para o desenvolvimento dos serviços prestados; b) parada de funcionamento dos setores dependentes do sistema, causando atraso nas atividades; c) atividades desenvolvidas de maneira duplicada; d) descrédito das informações fornecidas pelo sistema; e) comunicação de informação errônea; f) má qualidade no atendimento dos pacientes g) demora na tomada de decisão.

A falta de clareza das informações que devem compor um sistema corrobora com a deficiência da prestação dos serviços já que, por exemplo, no nível operacional, os funcionários não conseguem acompanhar atividades elementares com rapidez, ou seja, realizar reservas de leitos; verificar o estoque de medicamentos da farmácia; acompanhar os medicamentos a serem comprados. E, consequentemente, pelo fato de as informações do sistema do nível operacional "alimentarem" o sistema do nível gerencial, não é possível extrair informações sobre as atividades de monitoração e controle que possam apoiar o processo de tomada de decisão no nível gerencial.

Uma maneira de amenizar os problemas enfrentados por hospitais em relação ao processamento e organização das informações gerenciais é por meio de alterações no modo de operacionalização das atividades, incorporando um sistema de informação gerencial que possa apoiar as atividades-chave do hospital.

Para tanto, a adoção de um sistema de informação gerencial integrando as atividades hospitalares pode resultar em melhor controle dos procedimentos realizados, agilizando e apoiando a tomada de decisão. $\bigcirc$ uso de um sistema integrado de gestão - sistema de planejamento de recursos empresariais - enterprise resource planning (ERP), possibilita também a integração das atividades transfuncionais em diferentes níveis organizacionais.

Segundo Laudon e Laudon (2004), o sistema integrado de gestão coleta dados dos principais processos de negócio e os armazena em um único arquivo de dados abrangente (base de dados única), os quais podem ser utilizados por outras áreas da organização. Dessa forma, o gestor dispõe de informações mais precisas para administrar as operações diárias da organização, além de uma visão mais abrangente do fluxo de informação entre as áreas internas à organização.

No hospital, um sistema integrado de gestão poderia envolver, por exemplo, informações das áreas de atendimento aos pacientes: clínica médica, diagnóstico, terapia, materiais, financeira e serviço de apoio. Assim, integrando os dados desde o atendimento de pacientes até o faturamento das contas, seria possível monitorar e otimizar as atividades hospitalares.

Com esse tipo de sistema, os funcionários das diversas áreas poderiam acessar informações atualizadas em tempo real, ter acesso a relatórios, planilhas de custo e outros, melhorando a execução tanto das atividades operacionais quanto gerenciais e oferecendo melhor assistência aos pacientes.

Um Sistema de Informações Gerenciais (SIG) pode ser constituído de informações sobre os processos de negócio hospitalar abrangendo, por exemplo, os processos referentes à: internação, transferência e alta/ óbito; abertura de conta hospitalar; administração da ocupação de leito; reserva de leito e controle de lista de espera; controle de movimentação dos pacientes; controle de cauções e controle de pendências de atendimentos.

Um Sistema de Informações Gerenciais pode adquirir seus dados do Sistema de Processamento de Transações (SPT) como ilustrado na Figura 1 : 


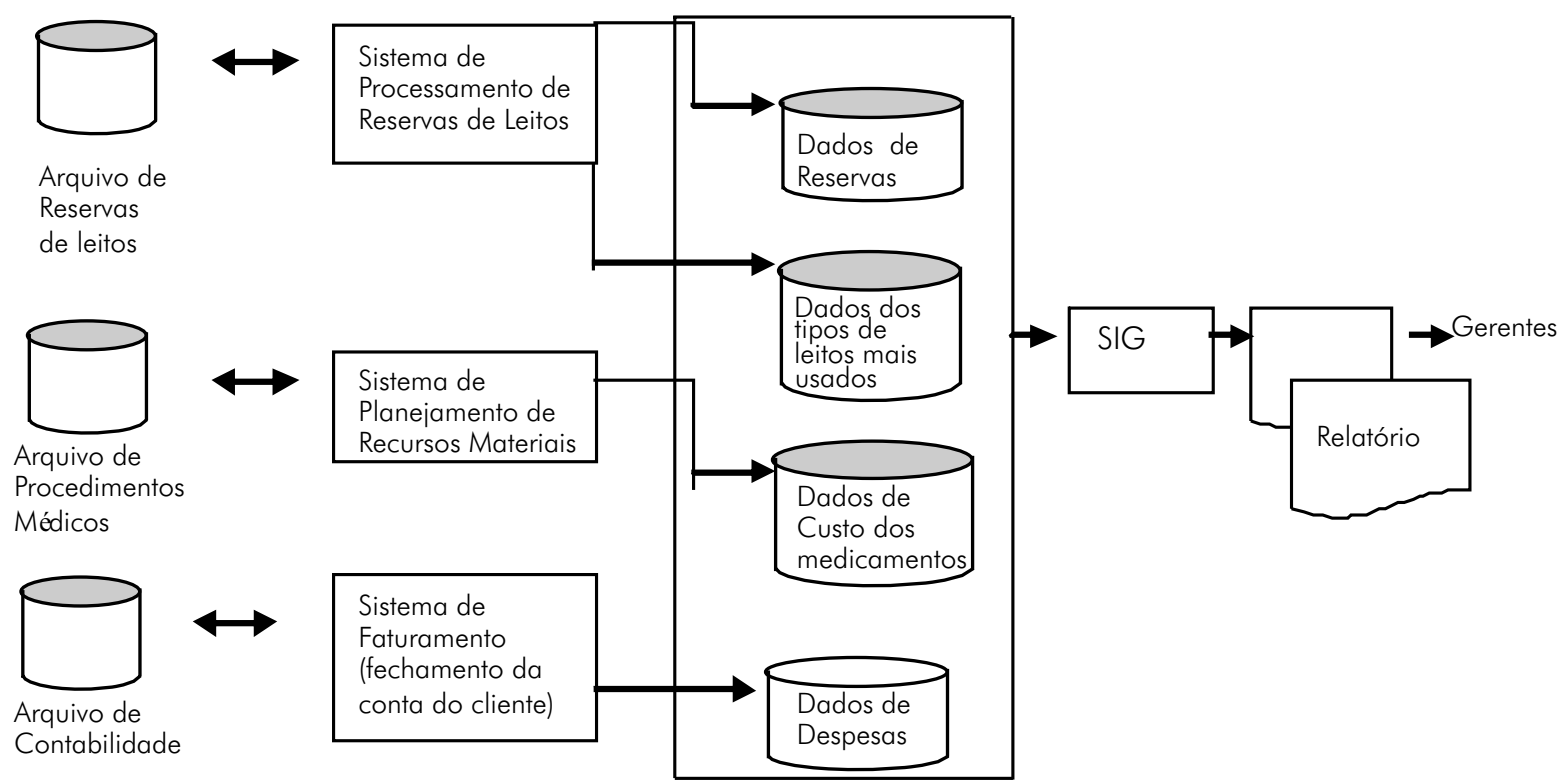

Figura 1. Como os sistemas de informações gerenciais adquirem seus dados do SPT da empresa.

Fonte: Adaptado de Laudon; Laudon, 2004, p.45.

Os dados resumidos obtidos dos três arquivos do Sistema de Processamento de Transações: reservas de leitos; procedimentos médicos e contabilidade são comprimidos e apresentados em relatórios para os gerentes, os quais são produzidos pelo Sistema de Informações Gerenciais. A Figura 2 ilustra o relatório que poderia ser produzido pelo SIG utilizando os arquivos do SPT mencionados acima:

Relatório consolidado por paciente segundo procedimentos médicos realizados em 2007

\begin{tabular}{ccccccc}
\hline $\begin{array}{c}\text { Código do } \\
\text { paciente }\end{array}$ & $\begin{array}{c}\text { Data do } \\
\text { Atendimento }\end{array}$ & $\begin{array}{c}\text { Descrição dos } \\
\text { Procedimentos Médicos }\end{array}$ & $\begin{array}{c}\text { Quantidade } \\
\text { realizada }\end{array}$ & $\begin{array}{c}\text { Materiais e Equipamentos } \\
\text { utilizados }\end{array}$ & $\begin{array}{c}\text { Custo } \\
\text { unitário }\end{array}$ & $\begin{array}{c}\text { Custo } \\
\text { total }\end{array}$ \\
\hline $2634 / 07$ & $24 / 02 / 2007$ & Ultrassonografia & 1 & Máuina XYZ & 1486 & 1486 \\
$2634 / 07$ & $28 / 4 / 2007$ & Doppler & 1 & Equipamento A & 850 & 850 \\
$2745 / 07$ & $27 / 3 / 2007$ & Ressonância Magmética & 2 & Aparelho ABC & 2389 & 4778 \\
\hline
\end{tabular}

Figura 2. Um exemplo de relatório que poderia ser produzido pelo SIG. Fonte: Elaboração dos autores.

O relatório da Figura 2 permite ao tomador de decisão comparar os procedimentos médicos que foram realizados com cada paciente em um determinado período, apoiando o processo de acompanhamento das enfermidades do paciente.
Além disso, mostra o custo total dos procedimentos médicos realizados a serem desembolsados pelo paciente. Em geral, os SIGs usam rotinas simples, como resumos e comparações, ao invés de modelos matemáticos ou técnicas estatísticas. 


\section{CONSIDERAÇÕES FINAIS}

A determinação das informações necessárias para apoiar a tomada de decisão é indubitavelmente essencial para que a organização defina o tipo de sistema a ser utilizado. Acredita-se que os sistemas formais de informação devem ser projetados após a definição das informações necessárias para acompanhar as atividades de uma área de. Sob este olhar, o sistema deve adaptar-se às necessidades de informação da organização e não o contrário.

O uso de um Sistema de Informações Gerenciais em hospitais corrobora com a gestão das informações

\section{REFERÊNCIAS}

DAVENPORT, T.H. Ecologia da informação: por que só a tecnologia não basta para o sucesso na era da informação. São Paulo: Futura, 1998.

HAWKINS, D.T. Information science abstracts:tracking the literature of information science. Part 1: Definition and map. Journal of American Society for Information Science and Technology, v. 52, n. 1, p. $44-53,2001$.

LAUDON,K.C.; LAUDON, J.P. Sistemas de informação gerenciais: administrando a empresa digital. São Paulo: Prentice Hall, 2004.

LE COADIC, Y.F. A ciência da informação. Brasília: Briquet de Lemos Livros, 1996. Tradução de: La science de l'information. excluir

OLIVEIRA, D.P.R. Sistemas de informações gerenciais. 7. ed. São Paulo: Atlas, 2001. no momento em que fornecem respostas a perguntas rotineiras que foram especificadas anteriormente e cujo procedimento de obtenção é pré-denifido. Os relatórios de um SIG possibilitam acompanhar e controlar os procedimentos realizados com os pacientes, além de agilizar a tomada de decisão.

A definição de informações que irá compor um sistema pode melhorar a prestação dos serviços, facilitando o acompanhamento das atividades elementares com maior rapidez, ao possibilitar, por exemplo, um melhor monitoramento do quadro clínico dos pacientes.
REZENDE, D.A.; ABREU, A.F. Tecnologia da informação aplicada a sistemas de informação empresariais: o papel estratégico da informação e dos sistemas de informação nas empresas. 3. ed. São Paulo: Atlas, 2003.

ROBREDO, J. Da ciência da informação revisitada: aos sistemas humanos de informação. Brasília, DF: Thesaurus; SSRR Informações, 2003.

SHANNON, C.E.; WEAVER, W. The mathematical theory of communication. Urbana: University of Illinois Press, 1962.

SOUZA, H.J.; VISELI, J.; SUGAHARA, C.R. Sistema de informação gerencial em hospitais. Revista Eletrônica de Gestão de Negócios, v.2, n.4, p. 73-87, 2006. 